\title{
Improved tumor imaging and therapy via i.v. IgG-mediated time-sequential modulation of neonatal Fc receptor
}

Jaspreet Singh Jaggi, ${ }^{1}$ Jorge A. Carrasquillo, ${ }^{2}$ Surya V. Seshan, ${ }^{3}$ Pat Zanzonico, ${ }^{2}$ Erik Henke, ${ }^{1}$ Andrew Nagel,2 Jazmin Schwartz, ${ }^{2}$ Brad Beattie, ${ }^{2}$ Barry J. Kappel,, Debjit Chattopadhyay, ${ }^{1}$ Jing Xiao, ${ }^{2}$ George Sgouros, ${ }^{4}$ Steven M. Larson,, ${ }^{1,2}$ and David A. Scheinberg 1

${ }^{1}$ Molecular Pharmacology and Chemistry Program and ${ }^{2}$ Department of Radiology, Memorial Sloan-Kettering Cancer Center, New York, New York, USA ${ }^{3}$ Department of Pathology, Weill Medical College of Cornell University, New York, New York, USA. ${ }^{4}$ Department of Radiology, Johns Hopkins University School of Medicine, Baltimore, Maryland, USA.

\begin{abstract}
The long plasma half-life of IgG, while allowing for enhanced tumor uptake of tumor-targeted IgG conjugates, also results in increased background activity and normal-tissue toxicity. Therefore, successful therapeutic uses of conjugated antibodies have been limited to the highly sensitive and readily accessible hematopoietic tumors. We report a therapeutic strategy to beneficially alter the pharmacokinetics of IgG antibodies via pharmacological inhibition of the neonatal Fc receptor ( FcRn) using high-dose IgG therapy. IgG-treated mice displayed enhanced blood and whole-body clearance of radioactivity, resulting in better tumor-to-blood image contrast and protection of normal tissue from radiation. Tumor uptake and the resultant therapeutic response was unaltered. Furthermore, we demonstrated the use of this approach for imaging of tumors in humans and discuss its potential applications in cancer imaging and therapy. The ability to reduce the serum persistence of conjugated IgG antibodies after their infusion can enhance their therapeutic index, resulting in improved therapeutic and diagnostic efficacy.
\end{abstract}

\section{Introduction}

Radiolabeled and toxin-conjugated monoclonal antibodies are becoming increasingly important for cancer imaging and therapy $(1,2)$. To date, 2 radiolabeled antibodies for the treatment of nonHodgkin lymphoma, 2 radiometal-labeled antibodies for imaging of colon and prostate cancer, and 1 drug-conjugated monoclonal antibody have been approved by the FDA. The blood half-life of intact IgG in humans is exceptionally long (23 days; refs. 3,4$)$. While this long serum persistence may allow for greater tumor targeting, it results in increased background activity and normaltissue toxicity. As a consequence of these unfavorable pharmacokinetics, successful therapeutic uses of the approved conjugated antibodies have been exclusively limited to the highly sensitive and readily accessible hematopoietic tumors.

The neonatal $\mathrm{Fc}$ receptor $(\mathrm{FcRn})$, a MHC class I-like protein highly expressed in neonatal rodent intestinal epithelium, human placental syncytioblasts, vascular endothelial cells, and hepatocytes, plays a central role in perinatal IgG transfer and protection of IgG from catabolism in adults (5-12). Genetic mutation or deletion of $\beta 2$ microglobulin, a component of the FcRn heterodimer, has been shown to result in reduced serum IgG concentration both in mice and in humans (13-16). The hypothesized mechanism of IgG protection by FcRn involves nonspecific pinocytosis of circulating IgG followed by low $\mathrm{pH}-$ dependent ( $\mathrm{pH} \sim 6.0$ ) binding of IgG to $\mathrm{FcRn}$ in the endocytic vesicles. The FcRn-IgG complex is transported away from the degradative lysosomal pathway to the cell surface, where $\operatorname{IgG}$ is

Nonstandard abbreviations used: BUN, blood urea nitrogen; CI, confidence interval; FcRn, neonatal Fc receptor; IVIG, i.v. IgG; RIC, radioimmunoconjugate. Conflict of interest: The authors have declared that no conflict of interest exists. Citation for this article: J. Clin. Invest. 117:2422-2430 (2007). doi:10.1172/JCI32226. released at the higher physiologic $\mathrm{pH}$ ( $\mathrm{pH} \sim 7.4)$. IgG not bound by FcRn is degraded in the lysosomes (17).

One way of altering the blood clearance of intact antibodies is by engineering mutations that affect their binding to $\mathrm{FcRn}$ (18-22). The conserved amino acid residues $\left(\mathrm{Ile}^{253}, \mathrm{His}^{310}\right.$, and $\mathrm{His}^{435}$ ) in the FcRn binding region of IgG are crucial for its binding to FcRn, and their genetic modification can prolong or shorten the blood half-life of IgG. However, this approach is not feasible as a general method to improve antibody pharmacokinetics because it reduces the delivery of the immunoconjugate to the tumor and also requires reengineering of each therapeutic or diagnostic antibody. In addition, the strategy is expensive and time-consuming, requiring regulatory approval for each modification. A more general and feasible approach would be to pharmacologically block the binding of the conjugated antibody to FcRn. We hypothesized that this could be accomplished via administration of high doses of IgG to competitively inhibit the binding of the conjugated antibody to the FcRn. Indeed, one proposed mechanism of the observed therapeutic benefit of high-dose i.v. IgG (IVIG) therapy in humoral autoimmune disease is the enhanced catabolism of pathogenic autoantibodies via FcRn blockade (23-26).

Here, we determined the mechanism and assessed the effectiveness of high-dose polyclonal IgG therapy in altering the pharmacokinetics of several different radiolabeled IgG antibodies in mice. We found enhanced blood and whole-body clearance of radioactivity, resulting in better tumor-to-blood image contrast and reduced normal-tissue radiation dose without compromising the targeting to the tumor or the resultant therapeutic response. Lastly, we demonstrated in humans the use of high-dose IgG therapy for tumor imaging, and we discuss its potential applications in imaging and therapy of cancer with conjugated antibodies. 

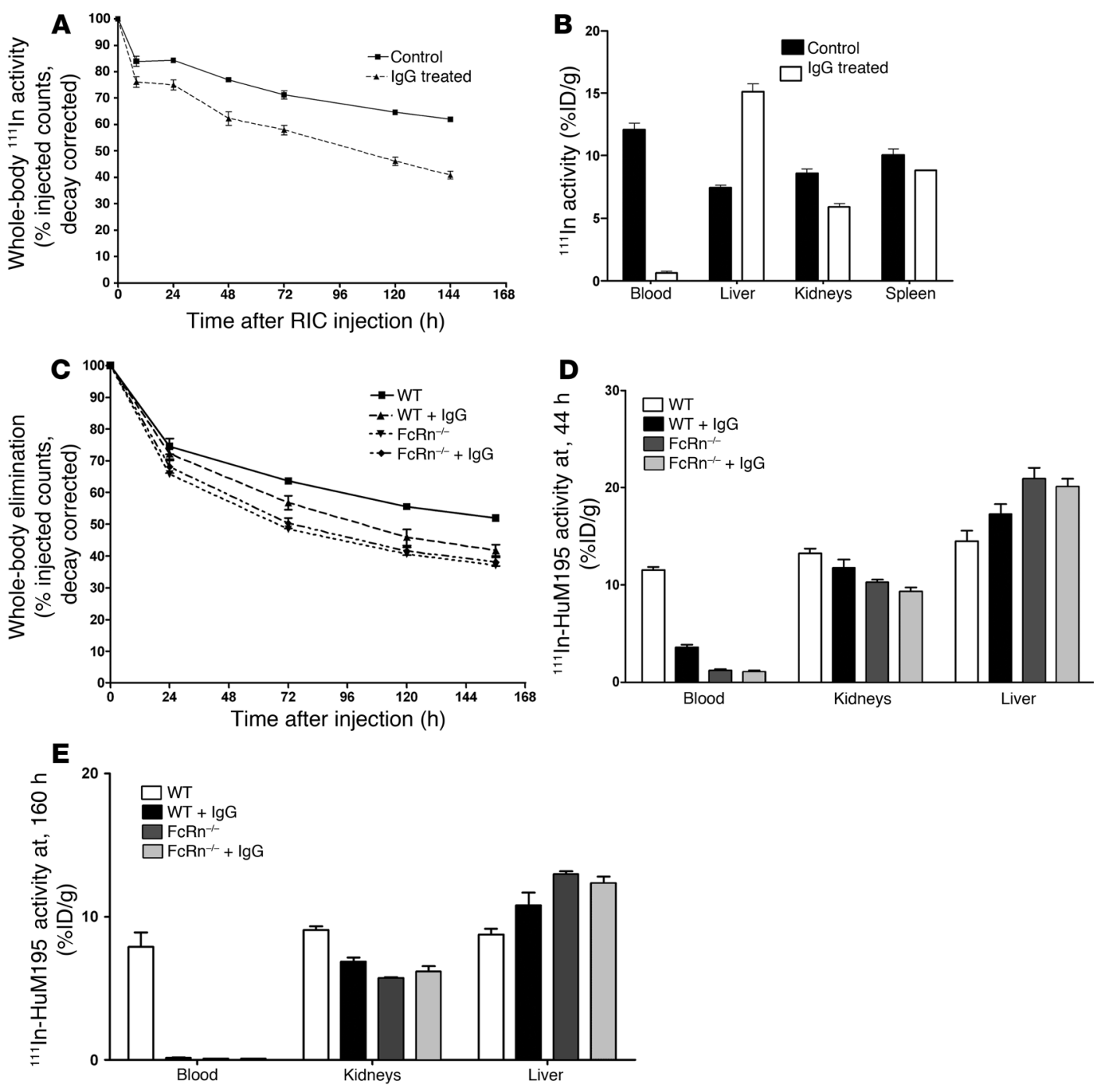

Figure 1

FcRn blockade enhances the clearance of radiolabeled IgG antibody. (A) Whole-body ${ }^{111}$ In activity at various time points after injection with ${ }^{111} \mathrm{In}$-HuM195 antibody ( $n=4$ per group). IgG-treated mice had faster whole-body clearance of radioactivity. (B) Organ distribution of ${ }^{111} \mathrm{In}$ activity in the same mice after the last whole-body measurement (144 hours) revealed accelerated blood clearance after IgG treatment. \%ID/g, percentage of injected dose per gram. (C-E) Whole-body and organ distribution of ${ }^{111}$ In activity in wild-type and FcRn knockout mice with or without IgG treatment. Genetic absence of a functional FcRn, or its pharmacological inhibition by high-dose IgG administration, resulted in accelerated whole-body elimination (C) and blood clearance of ${ }^{111} \mathrm{In}$-HuM195 antibody at 44 hours (D) and 160 hours (E) after injection. IgG treatment (1 g/kg i.p.; administered simultaneously with $\left.{ }^{111} \mathrm{In}-\mathrm{HuM} 195\right)$ did not alter whole-body clearance or organ distribution of ${ }^{111} \mathrm{In}$ in FcRn knockout mice. Data are mean \pm SEM. Scale of $y$ axes varies in $\mathbf{D}$ and $\mathbf{E}$.

\section{Results}

FcRn blockade enhances the clearance of radiolabeled IgG antibody. $\mathrm{BALB} / \mathrm{c}$ mice were injected i.v. with $2 \mu \mathrm{Ci}$ of indium-111-labeled ( ${ }^{111}$ In-labeled) humanized anti-CD33 IgG1 HuM195 antibody and simultaneously received either saline or $1 \mathrm{~g} / \mathrm{kg}$ polyclonal human IgG i.p. ( $n=4$ per group). Enhanced whole-body clearance of radioactivity was observed in IgG-treated mice relative to the control group (Figure 1A). Mice were sacrificed 148 hours after injection with the radioimmunoconjugate (RIC) to measure the organ distribution of ${ }^{111}$ In activity (Figure $1 \mathrm{~B}$ ). Postmortem biodistribution revealed substantially lower ${ }^{111}$ In activity in blood $(0.66 \pm 0.11$ versus $12.12 \pm 0.48 ; P<0.0001)$, as well as modest reductions in kidneys $(5.91 \pm 0.31$ versus $8.63 \pm 0.33 ; P=0.001)$ and spleen
$(8.83 \pm 0.05$ versus $10.07 \pm 0.49 ; P=0.04)$ of IgG-treated mice relative to untreated controls. The liver ${ }^{111}$ In activity, however, was significantly greater in the IgG-treated mice (15.14 \pm 0.61 versus $7.44 \pm 0.25 ; P<0.0001)$. To dissect the mechanism of the altered IgG pharmacokinetics, we performed a similar experiment using wild-type or FcRn-deficient (lacking the FcRn $\alpha$ chain; FcRn $n^{--}$) C57BL/6J mice. The whole-body elimination of radioactivity was faster in FcRn-deficient and IgG-treated wild-type mice than in untreated wild-type mice (Figure 1C). IgG administration, however, did not alter the whole-body clearance in FcRn-deficient mice. Postmortem biodistribution revealed enhanced blood clearance of radioactivity in FcRn-deficient and IgG-treated wild-type mice relative to untreated wild-type mice (Figure 1, D and E). IgG 

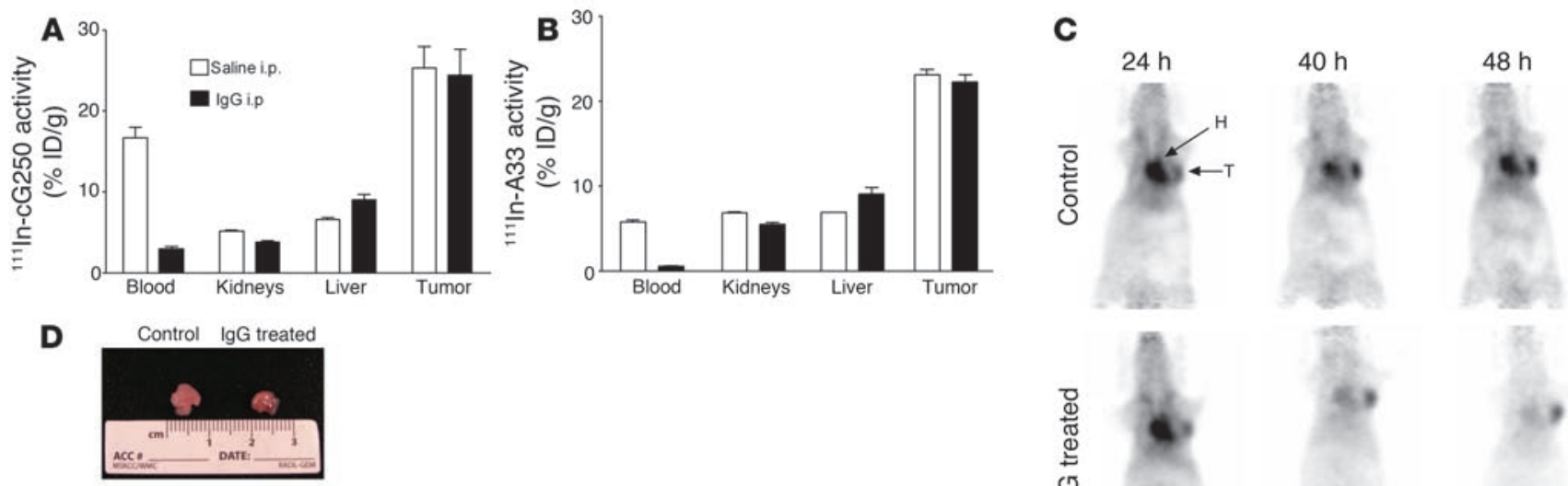

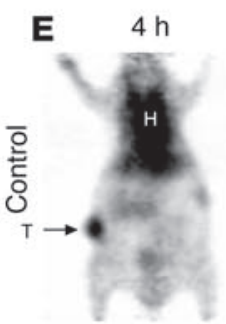

$18 \mathrm{~h}$
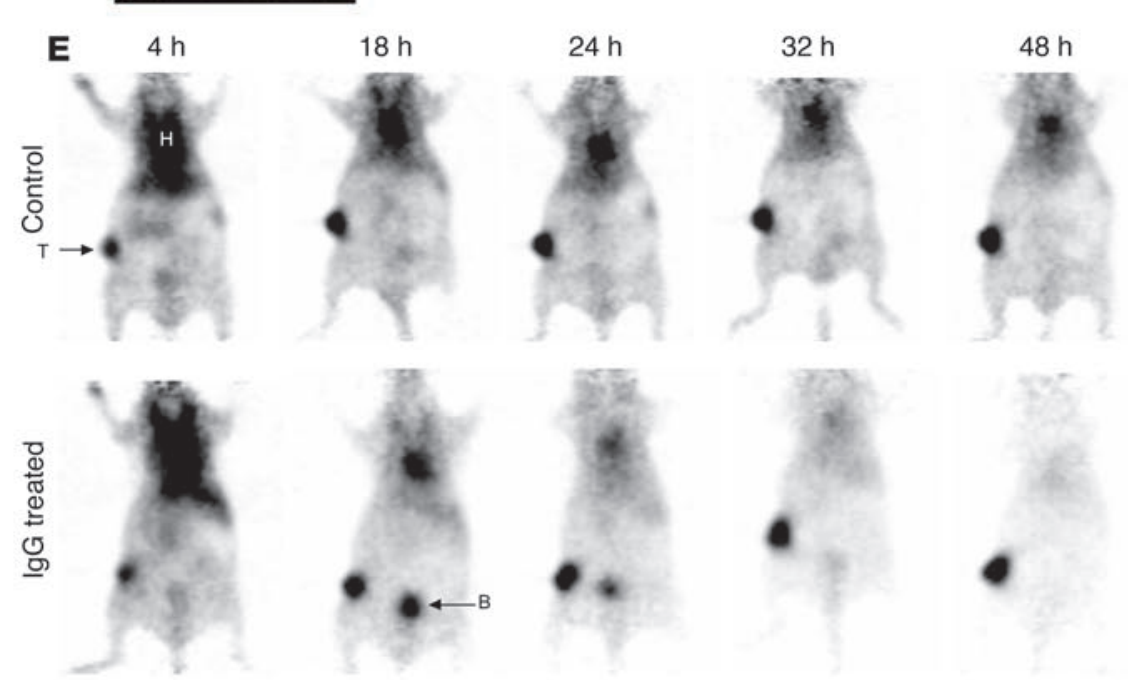

\section{Figure 2}

High-dose IgG administration enhances blood clearance and tumor-to-blood contrast in immuno-PET imaging without affecting the uptake of radiolabeled antibody in the tumor. (A and B) Organ distribution of ${ }^{111} \mathrm{In}$ activity at 72 hours after injection with ${ }^{111} \mathrm{In}-\mathrm{CG} 250$ (A) or ${ }^{111} \mathrm{In}-\mathrm{A} 33$ (B). IgG administration (1 g/kg i.p.; administered 24 hours after ${ }^{111} \mathrm{In}$-antibody) decreased the blood and renal ${ }^{111} \mathrm{In}$ activity without affecting the tumor uptake. Data are mean \pm SEM. (C and E) PET images of representative tumor xenograft-bearing mice at the indicated time points following injection with ${ }^{124}$-labeled cG250 (C) or A33 (E) antibody. (D) Excised tumors from mice in C. Enhanced tumor-to-blood contrast was seen in mice that received $1 \mathrm{~g} / \mathrm{kg}$ human $\mathrm{IgG}$, either 24 hours $(\mathbf{C})$ or 6 hours $(\mathrm{E})$ following ${ }^{124} \mathrm{l}$-antibody injection. $\mathrm{H}$, heart; $\mathrm{T}$, tumor; $\mathrm{B}$, urinary bladder.

administration enhanced the blood clearance of the radiolabeled antibody in wild-type mice at 44 hours (mean difference, 7.95; $95 \%$ confidence interval [CI], 6.74 to $9.15 ; P<0.001)$ and at 160 hours (mean difference, 7.77; 95\% CI, 5.33 to $10.22 ; P<0.001$ ), but not in FcRn-deficient mice at 44 hours (mean difference, 0.30; $95 \% \mathrm{CI},-0.90$ to $1.50 ; P>0.05$ ) or at 160 hours (mean difference, $0.001 ; 95 \% \mathrm{CI},-2.44$ to $2.45 ; P>0.05$ ), suggesting that the altered antibody pharmacokinetics seen with IgG therapy was mediated by this receptor.

High-dose IgG administration enhances tumor contrast in immunoPET imaging. With the foregoing evidence that blood clearance of radiolabeled antibodies was markedly accelerated with high-dose IgG administration, we hypothesized that this strategy would translate to contrast enhancement in imaging of tumors. SK-RC52 or SW1222 xenograft-bearing athymic mice were injected with ${ }^{111}$ In-labeled cG250 and A33 antibodies, respectively. High-dose $\operatorname{IgG}$ or saline was administered 24 hours after injection of the radiolabel to allow for tumor targeting of the radiolabeled antibody. Postmortem biodistribution 48 hours after high-dose IgG administration (Figure 2, A and B) showed 5- to 10-fold enhancement of the blood clearance of ${ }^{111}$ In-labeled antibodies (cG250, $16.72 \pm 1.24$ versus $3.0 \pm 0.26 ; P=0.0004 ; \mathrm{A} 33,5.83 \pm 0.18$ versus $0.59 \pm 0.04 ; P<0.0001)$ without affecting their tumor uptake (cG250, $25.25 \pm 2.70$ versus $24.35 \pm 3.22 ; P=0.84 ; \mathrm{A} 33,23.07 \pm 0.63$ versus $22.23 \pm 0.84 ; P=0.47)$.

For PET imaging, cG250 and A33 antibodies were labeled with iodine-124 ( ${ }^{124} \mathrm{I}$; a positron emitter), and high-dose IgG or saline was administered either 24 hours (for cG250 imaging) or 6 hours (for A33 imaging) after injection of the radiolabel. SK-RC-52 xenografts were visualized in mice by cG250 immuno-PET by 24 hours after injection of the radiolabeled antibody (Figure 2C). By 40 hours, both groups displayed comparable tumor uptake; however, IgG-treated mice showed markedly reduced cardiac activity (because of enhanced blood clearance) and increased activity in the urinary bladder. By 48 hours, IgG-treated mice had cleared the radioactivity from the blood while still retaining the tumor activity, resulting in enhanced tumor-to-blood contrast. The tumor in the IgG-treated mouse (Figure 2D), although smaller than that of 

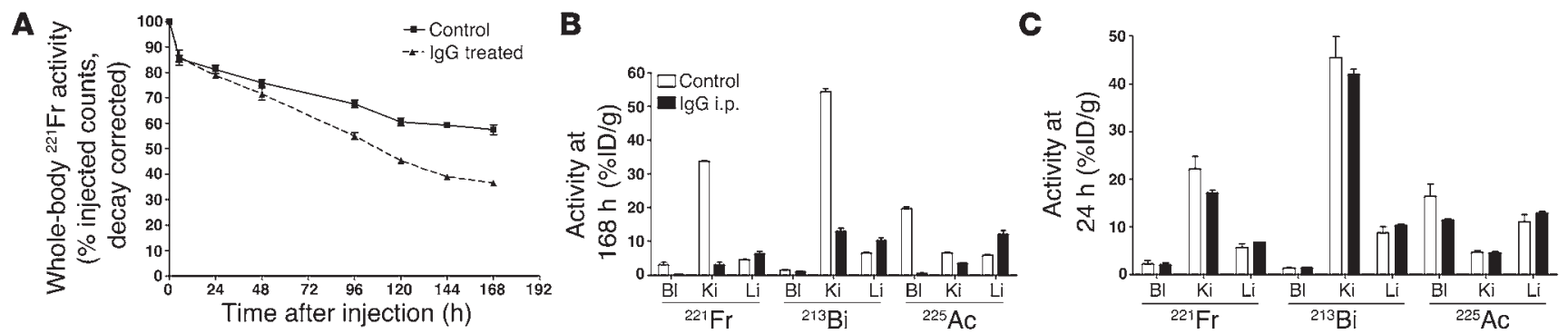

D

E

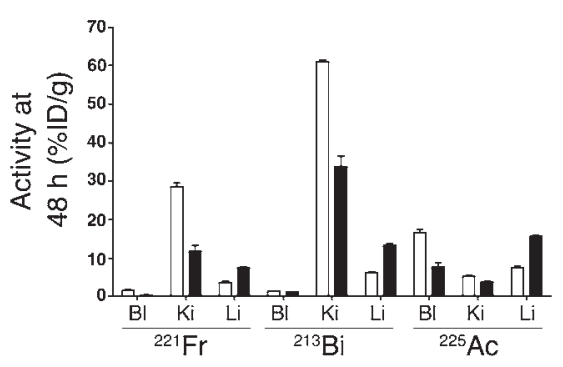

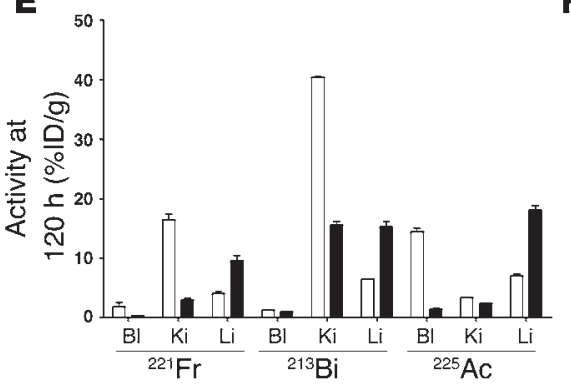

$F$

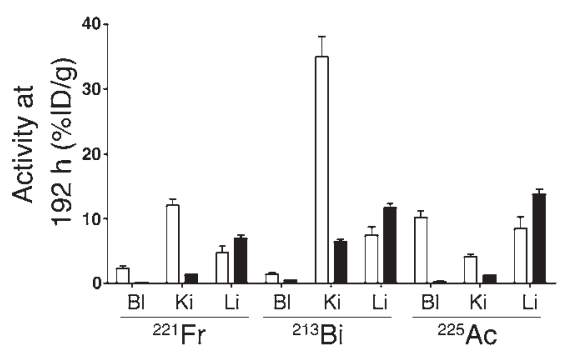

Figure 3

High-dose IgG administration alters the pharmacokinetics of the therapeutic ${ }^{225} \mathrm{Ac}$-labeled HuM195 antibody. (A) Whole-body ${ }^{221} \mathrm{Fr}$ activity (a decay product of ${ }^{225} \mathrm{Ac}$ ) at various time points after injection with ${ }^{225} \mathrm{Ac}$-HuM195 antibody ( $n=5$ per group). IgG-treated mice had faster wholebody clearance of radioactivity. (B) Organ distribution of ${ }^{225} \mathrm{Ac},{ }^{221} \mathrm{Fr}$, and ${ }^{213} \mathrm{Bi}$ activity in the same mice after the last whole-body measurement (168 hours). (C-F) Time course of the alteration of ${ }^{225} \mathrm{Ac}$-labeled HuM195 pharmacokinetics and that of ${ }^{225} \mathrm{Ac}$ decay elements ${ }^{221} \mathrm{Fr}$ and ${ }^{213} \mathrm{Bi}$ after high-dose IgG administration. IgG treatment (1 g/kg i.p.; administered at the same time as $\left.{ }^{225} \mathrm{Ac}-\mathrm{HuM} 195\right)$ resulted in enhanced blood clearance of ${ }^{225} \mathrm{Ac}-\mathrm{HuM} 195$ antibody at 24 (C), 48 (D), 120 (E), and 192 hours (F) after injection. Data are mean \pm SEM. Scale of $y$ axes varies in B-F. BI, blood; Ki, kidney; Li, liver.

the control mouse, was still readily discernible. The enhancement of tumor-to-blood contrast with high-dose IgG administration was even more pronounced in ${ }^{124} \mathrm{I}$-A33 imaging studies, in which tumors were visualized as early as 4 hours after injection (Figure 2E). At 18 hours after injection, IgG-treated mice displayed lower cardiac and higher urinary bladder activity, indicating enhanced blood clearance and metabolism and urinary elimination of radioactivity. By 48 hours after injection, although both groups showed similar tumor uptake, the IgG-treated mice displayed almost complete elimination of blood radioactivity and therefore enhanced tumor-to-blood contrast (Figure 2E and Supplemental Videos 1 and 2; supplemental material available online with this article; doi:10.1172/JCI32226DS1).

IgG administration alters the pharmacokinetics and absorbed radiation dose from therapeutic actinium-225 antibody constructs. We tested whether high-dose IgG could enhance the blood clearance of actinium$225-$ labeled ( ${ }^{225}$ Ac-labeled) antibody and as a result alter the biodistribution of the decay daughters of ${ }^{225} \mathrm{Ac}$. The $\alpha$ particle-emitting daughters, mainly francium-221 $\left({ }^{221} \mathrm{Fr}\right)$ and bismuth-213 $\left({ }^{213} \mathrm{Bi}\right)$, generated in vivo from circulating ${ }^{225} \mathrm{Ac}$-antibody constructs have the propensity to accumulate and irradiate the renal parenchyma (27) (Supplemental Figure 1). We hypothesized that high-dose IgG administration would enhance blood clearance and decrease the transit time of ${ }^{225} \mathrm{Ac}-\mathrm{HuM} 195$ in the blood. This would decrease the ${ }^{225} \mathrm{Ac}$ decays and therefore the generation of radioactive decay elements in the blood, resulting in decreased transport and accumulation of ${ }^{221} \mathrm{Fr}$ and ${ }^{213} \mathrm{Bi}$ in the kidneys. Mice were injected i.v. with $0.5 \mu \mathrm{Ci}{ }^{225}$ Ac-labeled HuM195 antibody with or without simultaneous high-dose IgG administration $(n=4)$. The whole-body clear- ance of radioactivity, determined via measurement of characteristic energy of the $\gamma$-photons of ${ }^{221} \mathrm{Fr}$, was enhanced by IgG administration (Figure 3A). Mice were then sacrificed at 170 hours after injection, and the tissue distribution of ${ }^{225} \mathrm{Ac},{ }^{221} \mathrm{Fr}$, and ${ }^{213} \mathrm{Bi}$ was determined (Figure 3B). IgG-treated mice revealed markedly lower ${ }^{225} \mathrm{Ac}$ activity in blood compared with control-treated mice (19.70 \pm 0.50 versus $0.6000 \pm 0.07 ; P<0.0001)$. As a consequence of the enhanced elimination of the radiolabeled antibody from blood, IgG-treated mice showed a large reduction in renal ${ }^{221} \mathrm{Fr}(33.70 \pm 0.21$ versus $3.02 \pm 0.67 ; P<0.0001)$ and ${ }^{213} \operatorname{Bi}(54.40 \pm 0.97$ versus $12.95 \pm 0.92$; $P<0.0001)$ activity. A marked decrease in blood ${ }^{225} \mathrm{Ac}$ and renal ${ }^{221} \mathrm{Fr}$ and ${ }^{213} \mathrm{Bi}$ activity was observed by 48 hours after injection, which became more pronounced at later time points (Figure 3, $\mathrm{C}-\mathrm{F}$, and Supplemental Table 1). The estimated absorbed dose to blood, kidneys, and liver of control mice was 155, 1,537, and 357.6 $\mathrm{Gy} / \mathrm{MBq}$, respectively. The radiation dose to blood, kidneys, and liver of IgG-treated mice was estimated to be 41.6, 306.8, and 678 $\mathrm{Gy} / \mathrm{MBq}$, respectively (Supplemental Table 2).

IgG administration protects against the toxicity of ${ }^{225} \mathrm{Ac}$ antibody constructs. Persistence of radiolabeled antibodies in blood results in increased toxicity (28). We investigated whether high-dose IgG administration could abrogate the early bone marrow and delayed renal toxicity in mice following a high dose of the cytotoxic ${ }^{225} \mathrm{Ac}-$ labeled HuM195 antibody. The early bone marrow toxicity was assessed by counting the wbc in peripheral blood. Twelve days after injection with $0.3 \mu \mathrm{Ci}$ or $0.6 \mu \mathrm{Ci}$ of ${ }^{225} \mathrm{Ac}$-labeled HuM195 antibody, injection of the RIC to mice caused a significant decline in the wbc count in their peripheral blood (Figure 4A). IgG treatment significantly reduced the bone marrow toxicity in mice that 

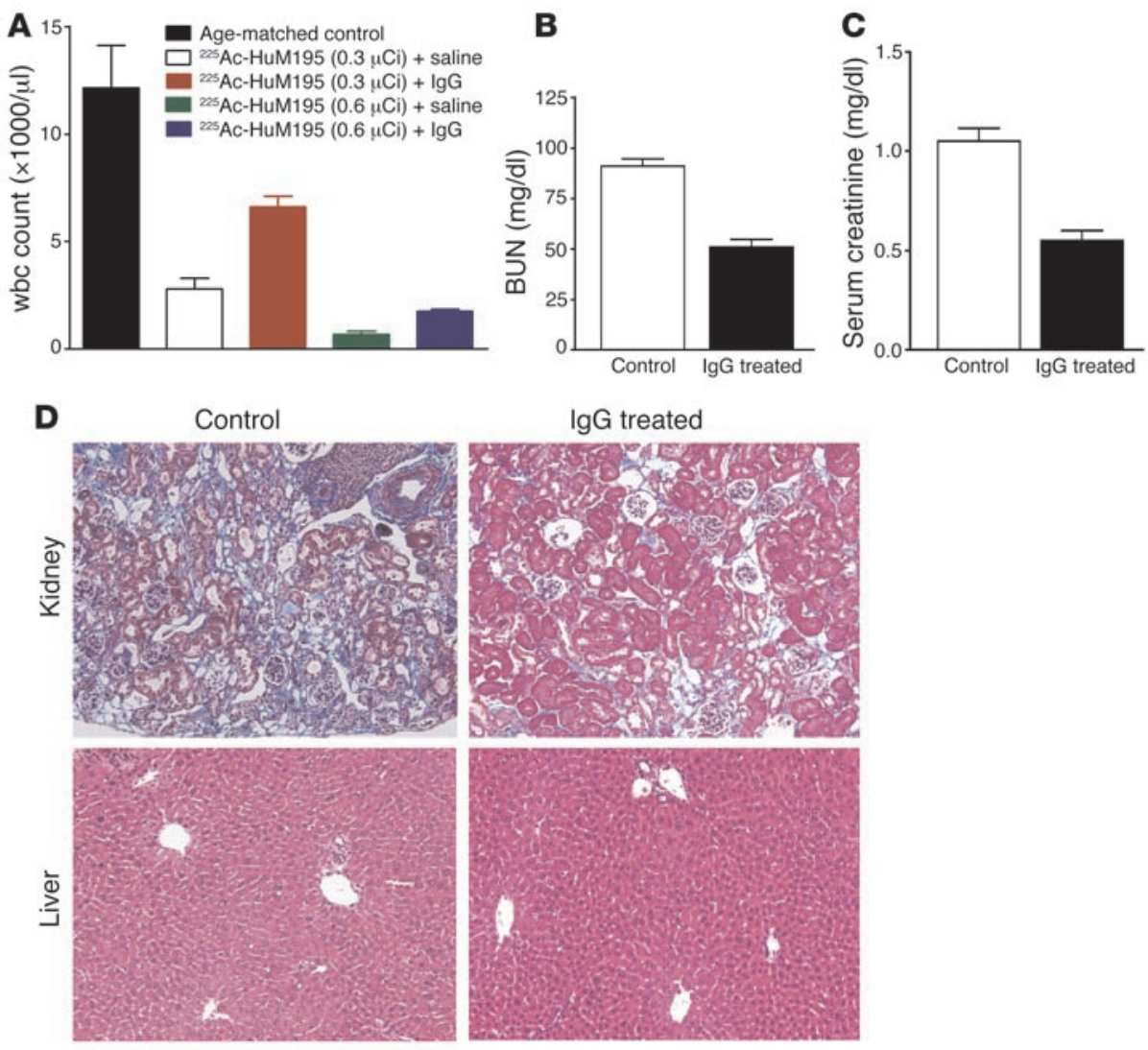

\section{Figure 4}

High-dose IgG protects animals against the toxicity of ${ }^{225} \mathrm{Ac}$ antibody construct. (A) Total leukocyte count 12 days after injection in mice injected with escalating doses of ${ }^{225} \mathrm{Ac}$-HuM195. IgG treatment was protective against the bone marrow toxicity of circulating ${ }^{225} \mathrm{Ac}-\mathrm{HuM} 195$ by enhancing its blood clearance. (B and C) Renal function of mice 35 weeks after injection with $500 \mathrm{nCi}{ }^{225} \mathrm{Ac}-\mathrm{HuM} 195$, with or without simultaneous IgG administration. (D) Kidney and liver histology 35 weeks after injection with $500 \mathrm{nCi}$ ${ }^{225} \mathrm{Ac}-\mathrm{HuM} 195$. IgG treatment $(1 \mathrm{~g} / \mathrm{kg}$ i.p.; administered at the same time as $\left.{ }^{225} \mathrm{Ac}-\mathrm{HuM} 195\right)$ protected mice against the late radiation-induced morphological and functional damage to the kidneys. No hepatic histopathology was seen in either group. Data are mean \pm SEM. received $0.3 \mu \mathrm{Ci}(P<0.01)$ but not $0.6 \mu \mathrm{Ci}(P>0.05)$ of ${ }^{225} \mathrm{Ac}$-labeled antibody. To test whether IgG therapy could mitigate the delayed radiation nephropathy, mice were injected with $0.5 \mu \mathrm{Ci}{ }^{225} \mathrm{Ac}$ labeled HuM195 and sacrificed 35 weeks after injection to assess the functional and histopathological damage to the kidneys. We have shown previously that $0.35 \mu \mathrm{Ci}{ }^{225} \mathrm{Ac}$-labeled HuM195 causes significant renal morphologic and functional damage at this time point (29). The blood urea nitrogen (BUN) and serum creatinine was significantly lower (Figure 4, B and C) in mice that received high-dose IgG (BUN, mean difference, $40.0 \pm 5.1 \mathrm{mg} / \mathrm{dl} ; 95 \% \mathrm{CI}$, 27.48 to $52.52 \mathrm{mg} / \mathrm{dl} ; P=0.0002$; serum creatinine, mean difference, $0.50 \pm 0.082 \mathrm{mg} / \mathrm{dl} ; 95 \% \mathrm{CI}, 0.30$ to $0.70 \mathrm{mg} / \mathrm{dl} ; P=0.0009$ ). Kidney sections from ${ }^{225} \mathrm{Ac}-\mathrm{HuM} 195$-injected control mice displayed diffuse subcapsular cortical atrophy, extensive tubulolysis with thickened and wrinkled basement membranes, glomerular crowding, and dilatation of the Bowman spaces (Figure 4D). The renal histopathologic damage seen in IgG-treated mice was significantly diminished in extent and severity, with only focal areas of tubulolysis and basement membrane thickening. Despite a greater radiation dose to the liver in IgG-treated mice, no significant morphologic damage was observed (neither group displayed histopathologic damage to the liver).

High-dose IgG administration prevents normal-tissue toxicity while preserving the therapentic effect. Having shown in separate studies that high-dose IgG administration significantly decreased the renal radiation dose and toxicity from ${ }^{225}$ Ac-labeled antibody via enhancement of its blood clearance and that the tumor uptake of the antibody was not significantly altered, we evaluated in a tumor therapy study whether we could protect against the doselimiting radiation nephropathy while preserving the antitumor efficacy. Mice that received 0.2 or $0.3 \mu \mathrm{Ci}{ }^{225} \mathrm{Ac}-\mathrm{A} 33$ had substantially inhibited tumor growth and enhanced survival (Figure 5, A and B). All but 2 mice in the group that received $0.2 \mu \mathrm{Ci}{ }^{225} \mathrm{Ac}-\mathrm{A} 33$ plus high-dose IgG and all but 1 mouse in the $0.2 \mu \mathrm{Ci}{ }^{225} \mathrm{Ac}-\mathrm{A} 33$ group were cured of their tumors, defined as having no detectable tumor at 140 days after implantation. All mice that received 0.3 $\mu \mathrm{Ci}{ }^{225} \mathrm{Ac}-\mathrm{A} 33$ (with or without IgG administration) were cured of tumors; these mice were sacrificed 30 weeks after tumor implantation, and their liver and kidney sections were examined by light microscopy. No significant liver pathology was observed in either group (Figure 5C). However, kidneys of mice that received $0.3 \mu \mathrm{Ci}$ ${ }^{225}$ Ac-A33 without IgG therapy displayed moderately severe radiation damage with focal areas of basement membrane thickening with tubulolysis. IgG-treated mice showed complete protection against this delayed radiation nephropathy.

${ }^{124}$ I-A33 immuno-PET imaging in patients that received high-dose IgG administration. Serial images and blood radioactivity levels were obtained in patients that received high-dose IgG 2 days after injection with ${ }^{124} \mathrm{I}-\mathrm{A} 33$. Initial images showed predominantly blood pool activity (Figure 6, A and B); however, significant blood clearance was seen at the 160 -hour time point, and clear visualization of A33 antigen-positive, metastatic aortocaval lymph node was observed (Figure 6, C-E). The A33 antigen positivity of the aortocaval node was confirmed by immunohistochemistry following surgical resection. A decrease in the blood radioactivity was seen following IVIG administration (Figure 6F).

\section{Discussion}

Monoclonal antibodies represent one of the fastest-growing classes of new therapeutic and diagnostic agents, with nearly 2 dozen FDA- 
A

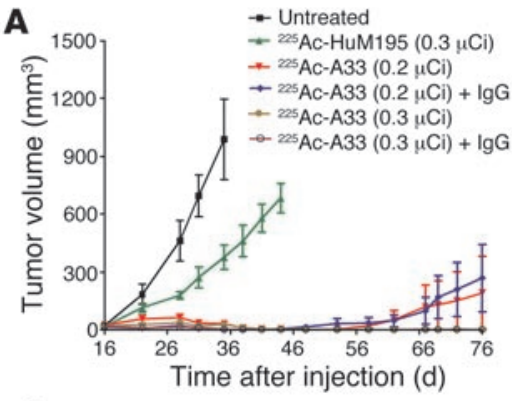

C

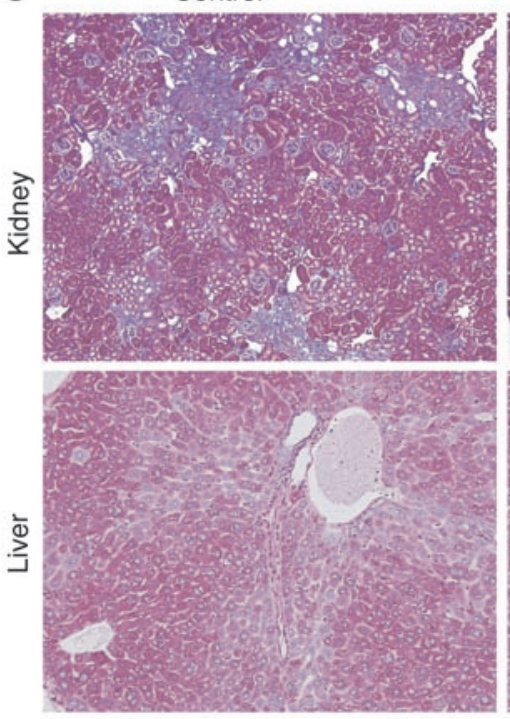

B

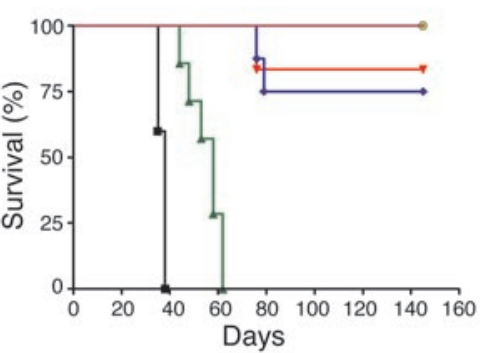

$\lg G$ treated

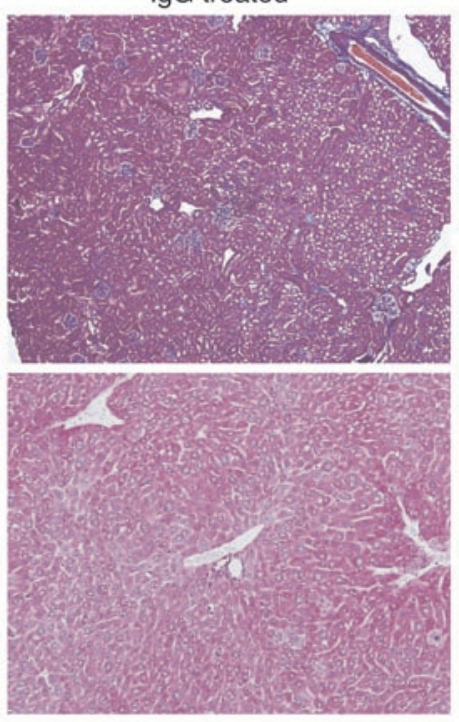

\section{Figure 5}

High-dose IgG protects animals against delayed radiation nephropathy while preserving the therapeutic effect of ${ }^{225} \mathrm{Ac}-\mathrm{A} 33$. (A) Tumor volume in various treatment groups of mice at the indicated time points. (B) KaplanMeier curve showing enhanced mouse survival following ${ }^{225}$ Ac-A33 treatment. A dose-dependent antitumor effect was seen in mice with ${ }^{225} \mathrm{Ac}-\mathrm{A} 33$ treatment. Data are mean \pm SEM. (C) Kidney and liver histology at 30 weeks after injection in mice that received $300 \mathrm{nCi}$ of ${ }^{225} \mathrm{Ac}-$ HuM195. IgG-treated mice were protected against the tubulolytic pathology seen in control mice. No hepatic histopathology was seen in either group. $\operatorname{lgG}(1 \mathrm{~g} / \mathrm{kg}$ i.p.) was administered 24 hours after ${ }^{225} \mathrm{Ac}-\mathrm{A} 33$.

and radiometal accumulation in these organs. Despite an estimated doubling of the radiation dose to the liver, no significant hepatic pathology was observed in IgG-treated animals. A possible explanation for this observation is the relative radioresistance of the liver (35). Our previous data in mice and nonhuman primates, in which high doses of ${ }^{225} \mathrm{Ac}$-HuM195 resulted in severe renal toxicity but no hepatic toxicity, are consistent with this finding $(29,36)$.

The biodistribution and whole-body clearance data in mice indicate that high-dose IgG therapy markedly increased the blood clearance of the radiometal-labeled antibody, which was followed by gradual elimination of the radioactivity from the liver and thus enhanced whole-body clearance. For whole-body clearance and postmortem biodistribution studies, radiometals $\left({ }^{111} \mathrm{In}\right.$ or ${ }^{225} \mathrm{Ac}$ ) were used to

approved antibodies for the treatment of cancer or inflammatory and autoimmune disorders now available. Most native therapeutic antibodies provide modest response rates against solid tumors, and strategies to improve potency typically involve the conjugation of a drug, toxin, or radioisotope to the antibody. However, this strategy does not markedly improve the therapeutic index because the slow clearance of monoclonal antibodies results in increased normal-tissue toxicity, typically to the bone marrow and secondarily to the kidneys, lungs or liver. The ability to accelerate the clearance and reduce the plasma half-life of IgG antibodies (several weeks in humans) after their infusion should improve their therapeutic indices and the resultant therapeutic and diagnostic efficacy.

The MHC-1, like FcRn, binds IgG and albumin at distinct sites and protects both proteins from catabolism (30). However, this protective capacity of $\mathrm{F} \mathrm{cRn}$ is saturable. It has been shown that the plasma half-life of IgG is inversely proportional to its serum concentration and that the plasma half-life of radiolabeled $\gamma$ globulin is shorter in patients with monoclonal gammopathy $(31,32)$. Because it is FDA approved and large amounts are readily available, we used human polyclonal IgG to pharmacologically inhibit the murine $\mathrm{FcRn}$, which is promiscuous and can bind human IgG with high affinity (33).

Biodistribution studies in mice revealed enhanced blood clearance and a higher liver uptake of the radiolabeled antibody following high-dose IgG therapy. An enhancement in liver uptake was expected because it was previously reported that FcRn is expressed in hepatic endothelium and the hepatocytes $(5,12,34)$ and that blockade of FcRn should result in degradation of the radiolabeled IgG label antibodies instead of radioiodine because iodine can be rapidly released from tissues following endocytosis and catabolism of radioiodinated antibodies and may therefore lead to an overestimation of the normal-organ and whole-body clearance. For the same reason, although enhancement in liver uptake of radioactivity was seen following high-dose IgG administration in biodistribution experiments, no liver uptake was seen in PET images, which used ${ }^{124} \mathrm{I}$ as a radiolabel. For PET studies, in which enhanced tumor-to-blood contrast is desirable, ${ }^{124} \mathrm{I}$ provides a quantitative measure of the blood clearance and tumor targeting. We showed the versatility of this approach in altering the pharmacokinetics of IgG antibodies by testing 3 different radiolabeled antibodies, each of which has been used in patients with respective tumor types. ${ }^{225} \mathrm{Ac}-\mathrm{HuM} 195$, an antibody construct currently in human clinical trials for patients with advanced myeloid malignancies, does not have a suitable mouse tumor model. Therefore, biodistribution and imaging studies in tumor-bearing mice were performed with radiolabeled cG250 or A33 antibodies because of the availability of reliable mouse tumor xenograft models.

The plasma clearance of ${ }^{124} \mathrm{I}-\mathrm{A} 33$ in humans, although resulting in favorable image contrast, was not as pronounced as that seen in mice that received the same dose $(1 \mathrm{~g} / \mathrm{kg})$ of polyclonal human IgG. The blunted effect in humans may be explained not only by biologic differences, but also by the 8-fold higher affinity of human IgG for mouse FcRn than for human FcRn (37). Therefore, higher doses of IgG may be required in humans to achieve the effects seen in mice. Petkova, Roopenian, and colleagues have addressed this issue by generating mice that express human FcRn 
A

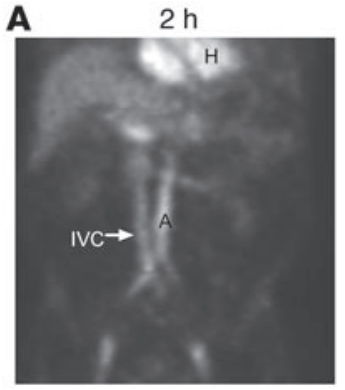

D $160 \mathrm{~h}$ CT image

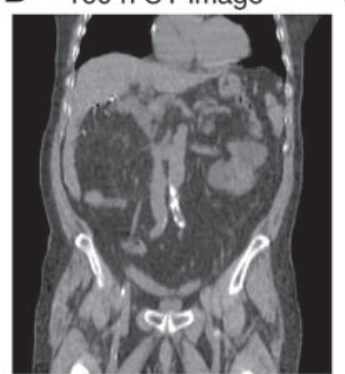

B

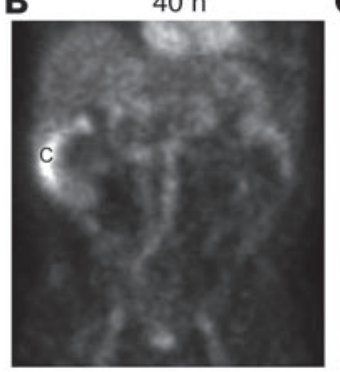

E

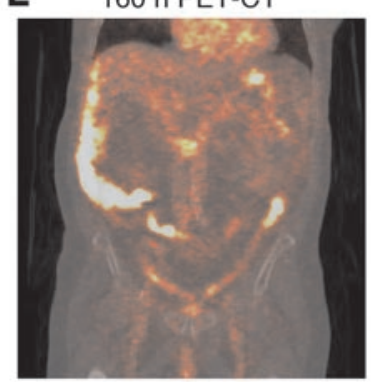

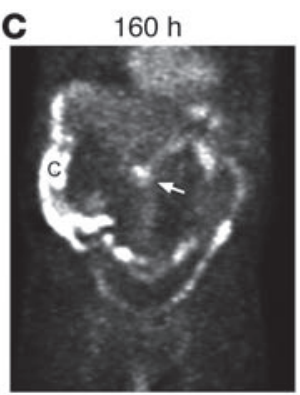

$\mathbf{F}$

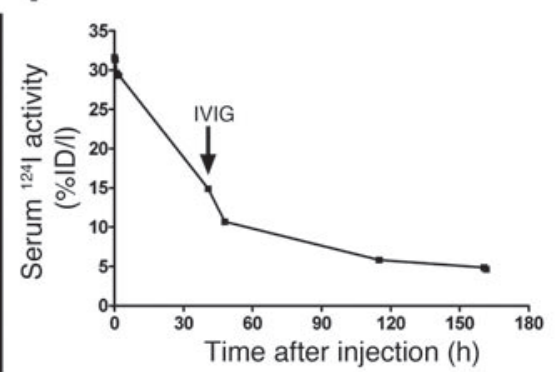

Figure 6

Immuno-PET imaging of colorectal cancer in patients with ${ }^{124}$ l-labeled A33 followed by high-dose IgG administration. (A) Early image (2 hours after ${ }^{124}$-A33 injection) of a representative patient shows the majority of radioactivity in the blood pool (heart and major vessels). (B) By 40 hours, in addition to the blood pool, uptake of radioactivity was seen in the colon. (C-E) Clearance of radioactivity from the blood pool at 160 hours allowed for visualization of the metastatic aortocaval lymph node (C, arrow), situated between the major blood vessels. The presence of tumor in the described lymph node was confirmed after surgical resection. (F) Serum ${ }^{124} \mid$ activity in the same patient at various time points after ${ }^{124} \mid-A 33$ injection, expressed as the percentage of injected dose per liter (\%ID/I). IVIG (1 g/kg) was administered to the patient 40 hours after ${ }^{124} \mathrm{I}-\mathrm{A} 33$, as shown. $\mathrm{H}$, heart; A, aorta; IVC, inferior vena cava; $\mathrm{C}$, colon.

(38), which should serve as a good surrogate for preclinical evaluation of diagnostic and therapeutic human IgG antibodies. The pharmacokinetic behavior of human IgG is also expected to differ in mouse and human systems. Moreover, the routes of administration of high-dose IgG were also different in mouse and human studies (i.p. and i.v., respectively). Plasma IgG concentrations of approximately 10 and $35 \mathrm{mg} / \mathrm{ml}$ in mice and humans, respectively, should suffice to functionally inhibit the ability of FcRn to protect IgG from catabolism (26). Based on basal plasma IgG concentration and its volume of distribution, 2 daily $1 \mathrm{~g} / \mathrm{kg}$ doses (the recommended therapeutic dose for autoimmune disorders) should result in a peak plasma IgG concentration of $40 \mathrm{mg} / \mathrm{ml}$ (39) and may be sufficient to saturate the FcRn in humans.

Previous studies have tried to mutate key amino acid residues in the IgG molecule that are critical for FcRn binding in order to generate antibodies with altered binding to $\mathrm{FcRn}(18,38,40)$. Although enhanced blood clearance of fragments with low affinity for FcRn was seen, their tumor uptake was also compromised (18). An important advantage of the high-dose IgG therapy described here, besides obviating the need for engineering each antibody, is that it allows for timing of the IgG administration to clear excess circulating antibody after tumor targeting has occurred. This is crucial, because the time required for optimal tumor targeting varies with each antigen-antibody-tumor system. For example, optimal tumor targeting in mice with A33 and cG250 was seen at 4 and 24 hours after injection, respectively, and therefore the $\operatorname{IgG}$ was administered to mice after 6 or 24 hours, respectively.
Another strategy, developed by Vaccaro et al., uses administration of engineered IgG antibodies with high affinity and reduced $\mathrm{pH}$-dependent binding to FcRn that are not readily released at the cell surface during exocytosis (40). As a consequence, low doses of engineered antibodies were required relative to wild-type polyclonal IgG to enhance blood clearance of endogenous antibodies. A relatively longer duration of action of this therapeutic via its inability to dissociate at the cell surface may be useful in autoimmune disorders, in which sustained degradation of endogenous pathogenic autoantibodies is desirable. However, for imaging or therapy with conjugated antibodies, in which a transient FcRn blockade to sufficiently accelerate the blood clearance of injected diagnostic or therapeutic antibody is desirable, an optimally timed high-dose IgG therapy is preferable.

In summary, we have shown that it is possible to effectively control the blood half-lives and therefore the therapeutic index of targeted IgG antibodies via pharmacological modulation of their interaction with the protective FcRn. The described approach resulted in enhanced tumor contrast and reduction of normal-tissue toxicity for tumor imaging and therapy.

\section{Methods}

Mice. Female BALB/c, C57BL/6J (wild-type as well as $\mathrm{FcRn^{-/ }}$ ), and athymic nude mice (4-8 weeks of age) were purchased from Taconic, The Jackson Laboratory, and the National Cancer Institute, respectively. All animal studies were conducted according to the NIH Guide for the care and use of laboratory animals and were approved by the Institutional Animal Care and Use committee at Memorial Sloan-Kettering Cancer Center.

Cells lines and xenografts. SW1222 and SK-RC52 cell lines (expressing A33 antigen and carbonic anhydrase IX, respectively) were cultured in RPMI medium supplemented with $10 \%$ fetal calf serum at $37^{\circ} \mathrm{C}$ in $5 \% \mathrm{CO}_{2}$. Xenografts were established in athymic nude mice by subcutaneous inoculation of $5 \times 10^{6}$ cells in $200 \mu \mathrm{l}$.

Preparation, quality control, and administration of RICs. Humanized antiCD33 IgG1 (HuM195) antibody was purchased from Protein Design Labs. Chimeric anti-carbonic anhydrase IX (cG250) and humanized anti-A33 antigen (huA33) were a gift from Ludwig Institute of Cancer Research. ${ }^{225} \mathrm{Ac}$ (Oak Ridge National Laboratory) and ${ }^{111}$ In (PerkinElmer) were conjugated to antibodies using a 2-step labeling method, as described previously $(41,42)$. Iodo-Gen method was used to radioiodinate antibodies with ${ }^{124} \mathrm{I}$ (18). Radiochemical purity and immunoreactivity of the RIC were determined as described previously (42). Mice were anesthetized with isoflurane and injected i.v. with the RIC in $100 \mu \mathrm{l}$. 
IgG treatment in mice. Mice were injected i.p. at indicated time points with $1 \mathrm{~g} / \mathrm{kg}$ purified polyclonal human IgG (Gammunex; Talecris) or an equal volume $(250 \mu \mathrm{l})$ of saline. In studies that evaluated the effect of high-dose IgG on pharmacokinetics and toxicity of radiolabeled antibodies in nontumor-bearing mice (Figures 1, 3, and 4), high-dose IgG was administered i.p. simultaneously with i.v. injection of the radiolabeled antibody. However, studies that tested the potential practical application of this strategy in terms of its effect on tumor targeting, imaging and therapy with radiolabeled antibodies, high-dose IgG administration was delayed by either 6 hours (imaging studies with ${ }^{124} \mathrm{I}-\mathrm{A} 33$ ) or 24 hours (imaging studies with ${ }^{124} \mathrm{I}-\mathrm{cG} 250$, biodistribution studies with ${ }^{111}$ In-labeled cG250 or A33, and therapy studies with ${ }^{225} \mathrm{Ac}-\mathrm{A} 33$ ) after injection of the radiolabeled antibody to allow for effective tumor targeting.

Estimation of whole-body radioactivity and organ distribution. Whole-body activity in mice was measured at various time points using a high-purity germanium detector (HPGe; Ortec) and expressed as percent of the injected dose (corrected for physical decay to the time of injection). The photopeaks at $218 \mathrm{keV}, 440 \mathrm{keV}$, and $171 \mathrm{keV}$ were used to assay ${ }^{221} \mathrm{Fr}$, ${ }^{213} \mathrm{Bi}$, and ${ }^{111} \mathrm{In}$ activity, respectively. For biodistribution studies, mice were sacrificed at the indicated time points after RIC injection (7-10 $\mu$ g antibody/mouse); their blood, kidneys, livers, and tumors (for some experiments) were harvested, weighed, and counted; and the percentage injected dose of activity per gram tissue was calculated, as described previously $(27,42)$.

${ }^{225} \mathrm{Ac}$-HuM195 toxicity studies in non-tumor-bearing mice. To evaluate the effectiveness of high-dose IgG in mitigating early bone marrow toxicity of ${ }^{225} \mathrm{Ac}$ labeled antibody, mice were injected with 0.3 or $0.6 \mu \mathrm{Ci}$ of ${ }^{225} \mathrm{Ac}-\mathrm{HuM} 195$ and injected simultaneously with either saline or $1 \mathrm{~g} / \mathrm{kg} \operatorname{IgG}$ i.p. Mice were euthanized 12 days after injection of the radiolabeled antibody, and their peripheral blood leukocyte count was measured. To study the effect of highdose IgG on delayed radiation nephropathy, mice were injected with $0.5 \mu \mathrm{Ci}$ ${ }^{225} \mathrm{Ac}$-HuM195 with simultaneous administration of either saline or $1 \mathrm{~g} / \mathrm{kg}$ IgG i.p., sacrificed 35 weeks after ${ }^{225} \mathrm{Ac}-\mathrm{HuM} 195$ injection, and evaluated for functional changes and histopathology in kidneys and liver.

Tumor therapy studies. Mice bearing SW1222 colon carcinoma xenografts were randomly assigned to various experimental groups that received either $1 \%$ human serum albumin (untreated; $n=5$ ), $0.3 \mu \mathrm{Ci}{ }^{225} \mathrm{Ac}$-labeled nonspecific $\mathrm{IgG}_{1}$ isotype antibody $\left({ }^{225} \mathrm{Ac}-\mathrm{HuM} 195 ; n=5\right), 0.2$ or $0.3 \mu \mathrm{Ci}$ ${ }^{225} \mathrm{Ac}$-labeled huA33 antibody $\left({ }^{225} \mathrm{Ac}-\mathrm{A} 33 ; n=7\right)$, or 0.2 or $0.3 \mu \mathrm{Ci}{ }^{225} \mathrm{Ac}$ labeled huA33 antibody along with $1 \mathrm{~g} / \mathrm{kg} \operatorname{IgG}(n=7)$ administered i.p. 24 hours after injection of the RIC. Treatments were administered 16 days after implantation of xenografts. Tumor size was measured with calipers, and tumor volume was calculated as $0.52 \times d_{1}{ }^{2} \times d_{2}$, where $d_{1}$ is the smaller diameter and $d_{2}$ is the larger diameter. Animals were followed long-term for survival advantage of the respective treatments. The mice that received $0.3 \mu \mathrm{Ci}{ }^{225} \mathrm{Ac}-\mathrm{A} 33$ with or without $1 \mathrm{~g} / \mathrm{kg}$ IgG were sacrificed 30 weeks after tumor implantation to evaluate the gross and histopathologic damage to the kidneys and liver.

Clinical and anatomic pathology. Blood was collected after mice were euthanized, and BUN and serum creatinine (LX 20 analyzer; Beckman Coulter) were determined. Liver and kidneys were harvested, fixed, processed, and paraffin-embedded. Sections $(2-3 \mu \mathrm{m})$ were stained with H\&E and Masson trichrome and examined with an Olympus BX45 light microscope.

Micro-PET imaging of mice. Tumor-bearing mice were injected i.v. with 250-280 $\mu$ Ci ${ }^{124}$ I-labeled cG250 or huA33 antibody. The antibody dose was $60-75 \mu \mathrm{g} /$ mouse. Normal thyroid uptake was blocked by administration of potassium iodide in drinking water. Mice were anesthetized with isoflurane and imaged with a Focus 120 microPET camera (Concorde Microsystems) at specified time points after injection. An energy window of 420-580 keV and a coincidence timing window of $6 \mathrm{~ns}$ was used. The resulting list-mode data were sorted into 2-dimensional histo- grams by Fourier rebinning, and transverse images were reconstructed in a $128 \times 128 \times 96$ matrix by filtered back-projection. The image data were corrected for nonuniformity of scanner response, dead-time count losses, the ${ }^{124} \mathrm{I}$ positron branching ratio, and physical decay to the time of injection. There was no correction applied for attenuation, scatter, or partialvolume averaging. The measured reconstructed spatial resolution of the Focus 120 is $\sim 1.6 \mathrm{~mm}$ full-width half maximum at the center of the field of view. The counts in the reconstructed images were converted to activity concentration (percent of injected dose per gram tissue) using a system calibration factor $(\mu \mathrm{Ci} / \mathrm{ml} / \mathrm{cps} /$ voxel) derived from imaging of a mousesize water-equivalent phantom. Region-of-interest analysis of the acquired images was performed using ASIPro software (Concorde Microsystems) for the tumor and major organs

Estimation of absorbed doses. The ${ }^{225} \mathrm{Ac},{ }^{221} \mathrm{Fr}$, and ${ }^{213} \mathrm{Bi}$ activity (percent of injected dose per gram) in blood, kidneys, and liver at various time points after injection with ${ }^{225} \mathrm{Ac}$-labeled HuM195 was used to estimate the absorbed dose to those organs, as described previously (27). The mean absorbed dose to the blood, kidneys, and liver was estimated by assuming that all $\alpha$ particle energy emitted by decays within each organ is completely absorbed within the organ.

Human immuno-PET studies. Human studies were reviewed and approved by the Institutional Review Board of Memorial Sloan-Kettering Cancer Center. All patients gave written informed consent following a thorough explanation of the study. Patients with colorectal cancer $(n=5)$ were injected with approximately $10 \mathrm{mCi} / 10 \mathrm{mg}{ }^{124} \mathrm{I}-\mathrm{A} 33$ i.v. in $30 \mathrm{ml}$ of $5 \%$ human serum albumin over 10 minutes. After 1-2 days, patients received IVIG $(1 \mathrm{~g} / \mathrm{kg}$ polyclonal human Ig). PET imaging of the abdomen and pelvis, using a GE Discovery PET/CT scanner, was carried out approximately 1-2 hours after administration of ${ }^{124} \mathrm{I}-\mathrm{A} 33$, before and after IVIG administration, and up to 3 hours prior to surgery. Image sets were reconstructed using standard iterative reconstruction techniques. Blood samples were collected at multiple time points between RIC administration and surgery, and the percent of injected dose per liter of serum was determined.

Statistics. Graphs were constructed using Prism (GraphPad Software). Statistical comparisons between the experimental groups were performed by either Student's $t$ test (2-group comparisons) or 1-way ANOVA with Bonferroni's multiple comparison post-hoc test (for comparison of 3 or more groups). All statistical comparisons were 2 -sided, and the level of statistical significance was set at $P<0.05$.

\section{Acknowledgments}

A33 and cG250 antibodies were a gift from the Ludwig Institute of Cancer Research. We thank Adam Boruchov and Chaitanya Divgi for helpful discussions. This work was supported by grants from the NIH (R01CA55349 and P01CA33049), the Doris Duke Foundation, the Ludwig Institute of Cancer Research, the Joseph LeRoy and Ann C. Warner Fund, and the William and Alice Goodwin Commonwealth Foundation.

Received for publication March 26, 2007, and accepted in revised form June 12, 2007.

Address correspondence to: David A. Scheinberg, Molecular Pharmacology and Chemistry Program, Memorial Sloan-Kettering Cancer Center, 415 East 68th Street, New York, New York 10021, USA. Phone: (646) 888-2190; Fax: (646) 422-0296; E-mail: d-scheinberg@ski.mskcc.org.

Jaspreet Singh Jaggi's present address is: Pfizer Global Research and Development, New London, Connecticut, USA. 
1. Milenic, D.E., Brady, E.D., and Brechbiel, M.W. 2004. Antibody-targeted radiation cancer therapy. Nat. Rev. Drug Discov. 3:488-499.

2. Verel, I., Visser, G.W., and van Dongen, G.A. 2005. The promise of immuno-PET in radioimmunotherapy. J. Nucl. Med. 46(Suppl. 1):164S-171S.

3. Spiegelberg, H.L., and Fishkin, B.G. 1972. The catabolism of human $\mathrm{G}$ immunoglobulins of different heavy chain subclasses. 3 . The catabolism of heavy chain disease proteins and of $\mathrm{Fc}_{\mathrm{C}}$ fragments of myeloma proteins. Clin. Exp. Immunol. 10:599-607.

4. Lobo, E.D., Hansen, R.J., and Balthasar, J.P. 2004. Antibody pharmacokinetics and pharmacodynamics. J. Pharm. Sci. 93:2645-2668.

5. Blumberg, R.S., et al. 1995. A major histocompatibility complex class I-related Fc receptor for IgG on rat hepatocytes. J. Clin. Invest. 95:2397-2402.

6. Borvak, J., et al. 1998. Functional expression of the MHC class I-related receptor, FcRn, in endothelial cells of mice. Int. Immunol. 10:1289-1298.

7. Junghans, R.P. 1997. Finally! The Brambell receptor (FcRB). Mediator of transmission of immunity and protection from catabolism for IgG. Immunol. Res. 16:29-57.

8. Gastinel, L.N., Ssimister, N.E., and Bjorkman, P.J. 1992. Expression and crystallization of a soluble and functional form of an Fc receptor related to class I histocompatibility molecules. Proc. Natl. Acad. Sci. U. S. A. 89:638-642.

9. Leach, J.L., et al. 1996. Isolation from human placenta of the IgG transporter, FcRn, and localization to the syncytiotrophoblast: implications for maternal-fetal antibody transport. J. Immunol. 157:3317-3322.

10. Simister, N.E., and Mostov, K.E. 1989. An Fc receptor structurally related to MHC class I antigens. Nature. 337:184-187.

11. Simister, N.E., Story, C.M., Chen, H.L., and Hunt, J.S. 1996. An IgG-transporting Fc receptor expressed in the syncytiotrophoblast of human placenta. Eur. J. Immunol. 26:1527-1531.

12. Telleman, P., and Junghans, R.P. 2000. The role of the Brambell receptor (FCRB) in liver: protection of endocytosed immunoglobulin $\mathrm{G}$ (IgG) from catabolism in hepatocytes rather than transport of IgG to bile. Immunology. 100:245-251.

13. Ghetie, V., et al. 1996. Abnormally short serum half-lives of IgG in beta 2-microglobulin-deficient mice. Eur. J. Immunol. 26:690-696.

14. Israel, E.J., Wilsker, D.F., Hayes, K.C., Schoenfeld, D., and Simister, N.E. 1996. Increased clearance of IgG in mice that lack beta 2-microglobulin: possible protective role of FcRn. Immunology. 89:573-578.
15. Roopenian, D.C., et al. 2003. The MHC class I-like IgG receptor controls perinatal IgG transport, IgG homeostasis, and fate of IgG-Fc-coupled drugs. J. Immunol. 170:3528-3533.

16. Wani, M.A., et al. 2006. Familial hypercatabolic hypoproteinemia caused by deficiency of the neonatal FC receptor, FcRn, due to a mutant beta2-microglobulin gene. Proc. Natl. Acad. Sci. U. S. A. 103:5084-5089.

17. Ober, R.J., Martinez, C., Vaccaro, C., Zhou, J., and Ward, E.S. 2004. Visualizing the site and dynamics of IgG salvage by the MHC class I-related receptor, FcRn. J. Immunol. 172:2021-2029.

18. Kenanova, V., et al. 2005. Tailoring the pharmacokinetics and positron emission tomography imaging properties of anti-carcinoembryonic antigen single-chain Fv-Fc antibody fragments. Cancer Res. 65:622-631.

19. Kim, J.K., et al. 1999. Mapping the site on human IgG for binding of the MHC class I-related receptor, FcRn. Eur. J. Immunol. 29:2819-2825.

20. Ghetie, V., et al. 1997. Increasing the serum persistence of an IgG fragment by random mutagenesis. Nat. Biotechnol. 15:637-640.

21. Dall'Acqua, W.F., et al. 2002. Increasing the affinity of a human IgG1 for the neonatal Fc receptor: biological consequences. J. Immunol. 169:5171-5180.

22. Medesan, C., Matesoi, D., Radu, C., Ghetie, V., and Ward, E.S. 1997. Delineation of the amino acid residues involved in transcytosis and catabolism of mouse IgG1. J. Immunol. 158:2211-2217.

23. Yu, Z, and Lennon, V.A. 1999. Mechanism of intravenous immune globulin therapy in antibodymediated autoimmune diseases. N. Engl. J. Med. 340:227-228

24. Hansen, R.J., and Balthasar, J.P. 2004. IVIG effects on autoantibody elimination. Allergy. 59:1124; author reply 1124 .

25. Hansen, R.J., and Balthasar, J.P. 2004. Mechanisms of IVIG action in immune thrombocytopenic purpura. Clin. Lab. 50:133-140.

26. Akilesh, S., et al. 2004. The MHC class I-like Fc receptor promotes humorally mediated autoimmune disease. J. Clin. Invest. 113:1328-1333. doi:10.1172/JCI200418838.

27. Jaggi, J.S., et al. 2005. Efforts to control the errant products of a targeted in vivo generator. Cancer Res. 65:4888-4895.

28. Wahl, R.L. 2005. Tositumomab and (131)I therapy in non-Hodgkin's lymphoma. J. Nucl. Med. 46(Suppl. 1):128S-140S.

29. Jaggi, J.S., et al. 2005. Renal tubulointerstitial changes after internal irradiation with alpha-particle-emitting actinium daughters. J. Am. Soc. Nephrol.
16:2677-2689.

30. Anderson, C.L., et al. 2006. Perspective-- FcRn transports albumin: relevance to immunology and medicine. Trends Immunol. 27:343-348.

31. Lippincott, S.W., et al. 1960. Turnover of labeled normal gamma globulin in multiple myeloma. J. Clin. Invest. 39:565-572.

32. Waldmann, T.A., and Strober, W. 1969. Metabolism of immunoglobulins. Prog. Allergy. 13:1-110.

33. Ober, R.J., Radu, C.G., Ghetie, V., and Ward, E.S. 2001. Differences in promiscuity for antibody-FcRn interactions across species: implications for therapeutic antibodies. Int. Immunol. 13:1551-1559.

34. Vriesendorp, H.M., and Vriesendorp, F.J. 2003. A review of the intravenous administration of radiolabeled immunoglobulin $G$ to cancer patients. High or low protein dose? Cancer Biother. Radiopharm. 18:35-46.

35. Bouvard, V., et al. 2000. Tissue and cell-specific expression of the p53-target genes: bax, fas, $\mathrm{mdm} 2$ and waf $1 / \mathrm{p} 21$, before and following ionising irradiation in mice. Oncogene. 19:649-660.

36. Miederer, M., et al. 2004. Pharmacokinetics, dosimetry, and toxicity of the targetable atomic generator, 225Ac-HuM195, in nonhuman primates. J. Nucl. Med. 45:129-137.

37. Zhou, J., Johnson, J.E., Ghetie, V., Ober, R.J., and Ward, E.S. 2003. Generation of mutated variants of the human form of the MHC class I-related receptor, FcRn, with increased affinity for mouse immunoglobulin G. J. Mol. Biol. 332:901-913.

38. Petkova, S.B., et al. 2006. Enhanced half-life of genetically engineered human IgG1 antibodies in a humanized FcRn mouse model: potential application in humorally mediated autoimmune disease. Int. Immunol. 18:1759-1769.

39. Bleeker, W.K., Teeling, J.L., and Hack, C.E. 2001. Accelerated autoantibody clearance by intravenous immunoglobulin therapy: studies in experimental models to determine the magnitude and time course of the effect. Blood. 98:3136-3142.

40. Vaccaro, C., Zhou, J., Ober, R.J., and Ward, E.S. 2005. Engineering the $F c$ region of immunoglobulin G to modulate in vivo antibody levels. Nat. Biotechnol. 23:1283-1288.

41. McDevitt, M.R., Ma, D., Simon, J., Frank, R.K., and Scheinberg, D.A. 2002. Design and synthesis of $225 \mathrm{Ac}$ radioimmunopharmaceuticals. Appl. Radiat. Isot. 57:841-847.

42. Borchardt, P.E., Yuan, R.R., Miederer, M., McDevitt, M.R., and Scheinberg, D.A. 2003. Targeted actinium-225 in vivo generators for therapy of ovarian cancer. Cancer Res. 63:5084-5090. 